\title{
Malondialdehyde and Homocysteine Levels in Patients with Unexplained Female Infertility
}

${ }^{1}$ Madhur Mahesh Gupta, ${ }^{2}$ Suresh Chari

\begin{abstract}
Objectives: To assess association in between malondialdehyde and homocysteine in females with unexplained infertility.
\end{abstract}

Methods: A case control study.

Setting: Hospital based.

Patients: Fifty females diagnosed with unexplained infertility were matched with fifty normal healthy controls.

Intervention: Blood samples for malondialdehyde, a lipid peroxidation product and homocysteine measurements.

Results: Our study suggests that females with unexplained infertility have increase in malondialdehyde along with hyperhomocysteinemia when compared with normal healthy controls.

Conclusion: Determination of levels of malondialdehyde and homocysteine should be incorporated as one of the factors for ascertaining the cause of female unexplained infertility.

Keywords: Malondialdehyde, Homocysteine, Unexplained infertility female.

How to cite this article: Gupta MM, Chari S. Malondialdehyde and Homocysteine Levels in Patients with Unexplained Female Infertility. J South Asian Feder Obst Gynae 2014;6(1):18-20.

Source of support: Nil

Conflict of interest: None

\section{INTRODUCTION}

L-Homocysteine (Hcy) is an endogenous amino acid, containing a free thiol group, which in healthy cells is involved in methionine and cysteine synthesis/resynthesis. About $80 \%$ of Hcy is bound to albumin (via a disulphide bond) in the plasma, where as the remaining $20 \%$ exists as free disulphides. Genetic factors, smoking, hypertension, serum creatinine, total cholesterol and protein and nutritional factors such as vitamin $\mathrm{B}_{6}, \mathrm{~B}_{12}$ and folate deficiency determine serum total homocysteine (tHcy) concentrations. Elevated circulating homocysteine or hyperhomocystei-

\footnotetext{
${ }^{1}$ Professor and Head, ${ }^{2}$ Professor

1,2Department of Biochemistry, NKP Salve Institute of Medical Sciences, Nagpur, Maharashtra, India

Corresponding Author: Madhur Mahesh Gupta, Professor and Head, Department of Biochemistry, NKP Salve Institute of Medical Sciences, Nagpur, Maharashtra, India, e-mail: drmadhur20@rediffmail.com
}

nemia (HHCY) is known to be associated with biotoxicity and has been underlined as an emerging risk factor for several diseases such as arterial and/or venous thrombosis ${ }^{1}$ and unexplained female infertility. ${ }^{2}$

In approximately 15 to $17 \%$ of couples, no reason for infertility is found and the infertility is defined as unexplained ${ }^{3}$ which is not a mere health problem but also a matter of social injustice and inequality.

Oxidative stress caused by the relentless formation of free radicals within an environment lacking proper antioxidant balance, is generated during oxidation of the free thiol group of Hcy. This mechanism is due to the binding of Hcy via a disulphide bridge with plasma proteins mainly albumin, or with other low-molecular plasma thiols or with a second Hcy molecule. Oxidation of Hcy may induce the subsequent oxidation of proteins, lipids and nucleic acids. ${ }^{4}$

This oxidative stress (OS) affect a variety of physiological processes such as folliculogenesis, oocyte maturation, endometrial cycle, luteolysis, implantation, and embryogenesis in the female reproductive tract thus influencing reproductive outcome. ${ }^{5,6}$

With this in mind it was thought worthwhile to evaluate and correlate the levels of malondialdehyde (MDA) a lipoperoxidation end product formed from reactive oxygen species (ROS) and homocysteine in females with unexplained infertility.

\section{MATERIALS AND METHODS}

A case control study approved by the Institutional Ethics Committee was carried out at NKP Salve Institute of Medical Sciences, Nagpur. Fifty females of unexplained infertility (group II) were included in the study. Unexplained infertility was diagnosed by the following criteria: Absence of male factor, i.e. normal semen analysis: Twenty million sperm per $\mathrm{ml}, \geq 50 \%$ forward motility, and greater than $40 \%$ normal morphology (using World Health Organization Criteria, 1999); adequate ovulation using either a mid-luteal serum progesterone greater than $10 \mathrm{ng} / \mathrm{ml}$, urine testing documenting the LH surge or serial transvaginal ultrasounds to monitor the development and rupture of a dominant ovarian follicle; normal FSH, LH, TSH, PRL; normal uterine cavity and patent tubes proved by hysterosalpingography or laparoscopy. These were age matched with 50 women 
Table 1: Levels of MDA and homocysteine in females with unexplained infertility

\begin{tabular}{lll}
\hline & Group I $(n=50)$ & Group II $(n=50)$ \\
\hline MDA $(\mathrm{nmol} / \mathrm{ml})$ & $1.76 \pm 0.20$ & $2.29 \pm 0.45^{*}$ \\
$\begin{array}{l}\text { Homocysteine } \\
(\mu \mathrm{mol} / \mathrm{l})\end{array}$ & $6.65 \pm 0.89$ & $13.24 \pm 5.27^{*}$ \\
\hline${ }^{*} \mathrm{p}<0.001$ when group II compared with group I
\end{tabular}

(group I control) with one or more successful pregnancy and without gestational complications (e.g. intrauterine growth restriction, still birth, abruptio placenta) and with any abortion. Women with anemia, gross pelvic pathology, uncontrolled chronic disease, history of pelvic inflammatory disease and those having male factor of infertility were excluded from the study. After having undergone detailed clinical history and physical examination, the infertile group underwent baseline investigations of infertility.

Five mililiter of fasting venous blood samples was collected in plain bulb from patients with infertility and normal healthy individuals.

Serum malondialdehyde (MDA), a marker of the oxidant status was determined by method of randox laboratory. This method was based on the fact that lipid peroxide condense with 1 methyl-2 phenyl indole (MPI) under acidic conditions resulting in the formation of a red chromophore. To determine specifically lipid peroxide in plasma, proteins are precipitated to remove water-soluble MPI reactive substance. The level of lipid peroxide is expressed in terms of malondialdehyde, which is unstable. Tetramethoxypropane, which is converted quantitatively to MDA in the reaction procedure, is used as standard.

For the measurement of homocysteine, all the specimens were transported to the laboratory within 30 minutes of collection. Thereafter, specimens were centrifuged for 5 to 7 minutes at $3000 \mathrm{rpm}$. The clear serum was transferred in a plastic vial and stored in refrigerator until analysis. Homocysteine was measured by microplate enzyme immunoassay kit method of Biorad laboratories.

Statistical analysis of difference was estimated using students ' $t$ ' test and correlation between variables was studied using pearson's correlation coefficient test.

\section{RESULTS}

As depicted in Table 1, MDA and homocysteine are significantly increased $(p<0.001)$ in females with unexplained infertility. Correlation analysis between MDA and homocysteine reveals that there is a positive correlation $(0.68, \mathrm{p}$ $<0.001)$ in females with unexplained infertility.

\section{DISCUSSION}

Homocysteine, a sulfur containing amino acid is an essential intermediate in the transfer of activated methyl groups in the activated methyl cycle. Normally plasma Hcy levels are decreased during pregnancy. Our study demonstrates an increase in the level of Hcy in females with unexplained infertility. HHcy may occur due genetic defect in enzyme involved in homocysteine metabolism like tetrahydrofolate reductase (MTHFR) and other deficiency in vitamin cofactors like folate and vitamin $\mathrm{B}_{12} \cdot{ }^{7}$ Under conditions of impaired folate status, the MTHFR 677 genotype encodes at thermolabile enzyme with reduced MTHFR activity and may cause moderate HHcy Since deficiency of MTHFR is rare and anemic patients were excluded from the study they being a cause for HHcy in infertility patients could be ruled out.

Apart from the procoagulant effect of Hcy, i.e. inhibition of the protein $\mathrm{C}$ activation, antithrombin III and thrombomodulin, it is related to an enhanced pro oxidant activity. Hcy modulates glutathione peroxidase expression, nitric oxide bioavailability and endothelin -1 production. ${ }^{8}$

It triggers accumulation of the intracellular ROS including $\mathrm{H}_{2} \mathrm{O}_{2}, \mathrm{O}_{2}^{-}$, and ${ }^{-} \mathrm{OH}$ through sulfur autooxidation. Moreover, sustained exposure to the endothelial cells to high levels of Hcy contribute to endothelial dysfunction. ${ }^{9}$ ROS oxygen species are known to modulate cellular functions resulting in a diseased cell. Our study suggests that ROS levels are increased in females with unexplained fertility. Research has revealed high levels of ROS in the peritoneal fluid specimen's from women with unexplained infertility in contrast to fertile controls undergoing tubal ligation. ${ }^{10}$ Normally, ROS may act as key signaling molecules in physiological processes but in excess are also known to mediate pathological processes. ${ }^{11}$ Numerous mechanisms have been proposed for the induction of infertility by OS: damage to the DNA of the oocytes and spermatozoa leading to defective fertilization; in case fertilization is achieved OS induce an apoptosis resulting in embryo fragmentation, implantation failure, abortion and impaired implantation. Moreover, OS may induce luteal regression and an insufficient luteal hormonal support for the continuation of pregnancy. ${ }^{12}$

Thus, from our study, Hcy and ROS may be involved in the pathogenesis of unexplained infertility. However, this being a case control study and both factors having a positive correlation it is difficult to assess whether Hcy or ROS singly or in association are responsible for the infertility.

Apart from the pivotal role of Hcy and ROS in infertility, ROS are said to be involved in the pathogenesis and Hcy has been described as an independent risk factor for cardiovascular disease. ${ }^{13}$ Endothelial cytotoxicity, lipid peroxidation, increased platelet adhesion, enhanced activation of the coagulation system and stimulation of vascular smooth muscle cell proliferation are the possible mechanisms which affect the vasculature. ${ }^{14}$ 
Hence, apart from other investigations for ascertaining the cause of female unexplained infertility, analysis of biochemical parameters like Hcy and ROS assay should be incorporated. Also, since Hcy and ROS are modifiable predictor of overall mortality and mortality due to cardiovascular causes, the role of homocysteine-lowering therapy and antioxidant supplementation in the treatment of such patients needs to be revisited.

\section{REFERENCES}

1. Martinelli I. Risk factors in venous thromboembolism. Thromb Haemost 2001;86:395-403.

2. D'Uva M, Di Micco P, Strina I, Alviggi C, Iannuzzo M, Ranieri A, Mollo A, De Placido G. Hyperhomocysteinemia in women with unexplained sterility or recurrent early pregnancy loss from Southern Italy: a preliminary report Thrombosis Journal 2007;5:10.

3. Akande AA, Idowu AA, Jimoh AK. Biochemical Infertility amoung females attending University of Ilorin teaching hospital, Nigeria. Nigerian J Clinical Practice March 2009;12(1):20-24.

4. Kalikiri PC. Hyperhomocysteinemia - A risk factor worth considering. JIACM 2003;4(2):147-151.

5. Sekhon LH, Gupta S, Kim Y, Agarwal A. Female Infertility and Antioxidants Current Women's Health Reviews 2010;6:84-95.

6. Agarwal A, Gupta S, Sikka S. The role of free radicals and antioxidants in reproduction Curr Opin Obstet Gynecol 2006; 18:325-332.
7. Lin PT, Huang MC, Lee BJ, Cheng CH, Tsai TP, Huang YC. High plasma homocysteine is associated with the risk of coronary artery disease independent of methylenetetrahydrofolate reductase 677C. T genotypes. Asia Pac J Clin Nutr 2008;17(2):330-338.

8. Rodriguez-Nieto S, Chavarria T, Martinez-Povedo B, Sanchez Jimenez F, Quesada AR, Medina MA. Anti-angiogenic effects of homocysteine on cultured endothelial cells. Biochemical and Biophysical Res Commun 2002;293:497-500.

9. Duan J, Xu Haishan, Dai S, Wang X, Wu Y, Zhang Y, Sun R, Ren J. Phytoestrogen $\alpha$-zearalanol inhibits homocysteine: induced endothelial-1 expression and oxidative stress in human umbilical vein endothelial cells. Atherosclerosis 2007;197:549-555.

10. Gupta S, Malhotra N, Sharma D, Chandra A, Agrawal A. Oxidative stress and its role in female infertility and assisted reproduction: clinical implications 2009;2(4):147-164.

11. da Silva FM, Marques A, Chaveiro A. Reactive oxygen species: A double-edged sword in reproduction. The open Veterinary Science Journal 2012;4:127-133.

12. Agrawal A, Gupta S, Sharma R. Oxidative stress and its implications in female infertility - a clinician perspective. Reproductive Biomedicine Online 2005;11(5):641-650.

13. Tarkun I, Cetinarsian B, Canturk Z, Turemen E. The plasma homocysteine concentrations and relationship with insulin resistance in young women with polycystic ovary syndrome. Turkish Journal of Endocrinology and Metabolism 2005;1: 23-28.

14. Tanrikulu-Kilic F, Bekpinar S, Orhan Unlucerci Y. Insulin resistance is not related to plasma homocysteine concentration in healthy premenopausal women. Physiol Res 2006;55:285-290. 